\title{
Computed Tomographic Diagnosis of Spinal Diseases in Dogs
}

\author{
S. Heera Banu* \\ Pallivasal Street, Thevaiyur (po), Perambalur, Tamil Nadu, India \\ *Corresponding author
}

\begin{abstract}
A B S T R A C T
Keywords

Computed

tomography, CT-

Myelography,

Spinal lesions, Dogs

Article Info

Accepted:

26 July 2018

Available Online:

10 September 2018

Computed Tomography is an noninvasive imaging method for diagnosis of vertebral and spinal cord lesions in small animals. There are retrospectively evaluated CT studies at nine dogs presented at Madras Veterinary College Teaching Hospital, Chennai with various degrees of neurological deficit and suspected with vertebral or spinal cord lesions. Out of these eight dogs, Different breeds including five Labrador retriever, one Daushand, one German shepherd and one Non-descriptive breed. All the dogs reported with neurological deficit were male. The mean age was 5.43 Yrs. CT studies were performed under general anesthesia, dorsal recumbency, without contrast medium in $4 \mathrm{dogs}$ and with contrast medium Iohexol $0.22 \mathrm{ml} / \mathrm{Kg}$ B.wt into subarachnoid space at occipito-atlantial junction (CT- Myelography) in 3 dogs. There were found one vertebral fracture, one vertebral fracture with luxation, three disc herniations, one extramedullary intradural tumor, one myelomalacia and one dural calcification. In conclusion CT is a valuable diagnostic tool for detection and characterization of spinal lesions.
\end{abstract}

\section{Introduction}

Vertebral column or spine of dog includes 7 cervical, 13 thoracic, 7 lumbar, 18-22 coccygeal vertebrae and the spinal cord have four longitudinal segments includes, cervical (C1-C5); cervical enlargement (C6-T2); thoraco-lumbar (T3-L3) and lumbar enlargement (L4-S2) (Evans et al., 1993). A disorder of each of these spinal cord segments result in a combination of neurological signs (Lecouteur, 2004). Common spinal disease in dogs based on the VITAMIN-D acronym includes vascular, inflammatory/ infectious, trauma, toxic, anomalous, metabolic, idiopathic, neoplastic, nutritional, degenerative/developmental disorders.
Intervertebral disc diseases, cervical spondylomyelopathy, vertebral fractures and luxations were commonly reported conditions which cause neurological deficit in dogs (Costa et al., 2010).

Survey radiography and myelography was the most commonly used to diagnose intervertebral disc diseases in veterinary practice but both methods having poor diagnostic value to localize and lateralize the IVD extrusion (Schulz et al., 1998). Conventional radiography was inadequate to diagnose the vertebral fracture and luxations with spinal cord compression in acute spinal trauma patients (Kinnes et al., 2006). In recent years, computed tomography a 
sensitive, non-invasive diagnostic and evaluating technique, has been used in veterinary practice (Jones an Inzana, 2000). Computed tomography is a fast and efficient to image canine spine (Olby et al., 2000). Computed tomography image provided clinically important information on anatomic structures and relationship between bony vertebral canal, meningeal spaces, spinal cord and intervertebral discs in three dimensional view which is not possible in conventional diagnostic modalities (Jones et al., 1994).

\section{Materials and Methods}

Out of the nine dogs, five were Labrador retriever, one was Daushand, one German shepherd and another one was non-descriptive breed. Six dogs were intact male and one was intact female. The mean age was 5.43 Yrs (10 months- 8 yrs). The mean weight was 29.3 Kgs (14-54 Kgs). Four dogs presented with paraplegia of pelvic limbs and the other three dogs were presented with tetraplegia.

Clinical examination, gait evaluation and neurological examinations includes cranial nerve reflexes, postural reactions (Wheel barrow, hopping, conscious proprioception, hemiwalking), spinal reflexes of thoracic limbs (Biceps, triceps, extensor carpi, flexor tendon and withdrawl), spinal reflexes of pelvic limbs (Patellar, gastrocnemius, cranial tibial, sciatic and withdrawal), panniculus reflex all were performed on all the animals at the time of presentation and localized the lesion (Palus, 2014). Plain radiographs of the localized vertebrae were taken in both lateral and ventrodorsal view to diagnose the bony lesions.

The CT studies were performed using, third generation GE 32 multislice Scanner. Before scanning procedure dogs premedicated with Atropine $0.04 \mathrm{mg} / \mathrm{Kg}$ Bwt followed by Xylazine $1.1 \mathrm{mg} / \mathrm{Kg} \mathrm{Bwt}$. Induction was done with Ketamine $5 \mathrm{mg} / \mathrm{Kg}$ Bwt + Diazepam $0.5 \mathrm{mg} / \mathrm{Kg} \mathrm{Bwt}$ and the maintenance done with the same if needed. During scanning procedure dogs were positioned under dorsal recumbency, thoracic limbes extended in a cranial direction and the pelvic limbs extended in a caudal direction (Jones et al., 1994). The scout radiograph was taken to confirm the position of the vertebral column then the area of interest was selected. The transverse images were taken at $1 \mathrm{~mm}$ slice thickness with the settings of $120 \mathrm{Kvp}$ and 90-160 mAs according to the size of the dog and the scan time was 2 second per slice. To visualize the bony vertebrae setting kept at bony window and for the spinal cord settings kept at soft tissue window level (Daraban et al., 2012). CT studies were performed without contrast medium in 4 dogs. CT myelography was done in 3 dogs. After a plain CT scanning, a contrast medium Iohexol $0.22 \mathrm{ml} / \mathrm{Kg}$ Bwt was injected in a subarachnoid space at occipito-atlantial junction.

All the transverse images were assessed for the presence of vertebral (or) spinal cord (or) intervertebral lesions.

\section{Results and Discussion}

On CT transverse sections bony part of spine includes vertebral body, pedicles, laminae, articular process, spinous process, transverse process and mammillary process were well visualized. Spinal cord is apparently round shaped surrounded by an epidural fat, its density is between $27-40 \mathrm{HU}$.

Out of these seven dogs one paraplegic dog had L1 vertebral fracture, one paraplegic dog had T8 fracture and luxation, two tetraplegic dogs had a disc herniation at C6 level, one paraplegic dog had a disc herniation at L5, L6 level and another one tetraplegic dog had extramedullary, intradural tumor at C6 level. 
The vertebral fractures are easily visualized without any contrast medium. Out of the two vertebral fracture, one was incomplete vertebral body and complete right pedicular fracture of L1 with significant spinal cord compression, another one was complete bilateral pedicular fracture and luxation of $\mathrm{T} 8$ along with significant spinal cord compression.

Intervertebral disc is observed as hypodense round structure between two vertebral endplates. Disc herniations are easily visible without any contrast medium but CT myelography helped in delineate the spinal cord and to know the degree of spinal cord compression. Out of the four disc herniation, two were at C6 level, two were at L5, L6 level with significant spinal cord compression. One chondrodystrophic dog had multiple Hansen type I disc herniation along with disc calcification at lumbar spinal cord segments. Other dogs had a disc herniation at single site.

Extramedullary, intradural spinal tumor was diagnosed at C6 level in one dog without contrast medium. The intradural tumor lesion was poorly visualised. In transverse section of CT myelography, the extramedullary intradural tumor was easily visualized at the ventral aspect of C6 spinal segment which was pushed the spinal cord dorsally along with spinal cord edema (Myelopathy) at C5, C7and T1 spinal cord segment.

Myelomalacia appeared as a hyperdense area within the spinal cord.

Computed tomography (CT) is an efficient three dimensional diagnostic tool to diagnose the spinal lesions than conventional radiography and myelography in dogs. CT myelography is giving better details about the degree of spinal cord compression and diagnosis of spinal cord tumors.

\section{References}

Costa, R. C and Moore, S.A. 2010. Differential diagnosis of spinal diseases. Vet Clin Small Anim. 40: 755-763.

Daraban, C., Vulpe, V., Bocaneti, F., Mennonia, G., Sacccone, M., Fatone, G. and Meomartino, L. 2012. CT findings of thoracolumbar spine lesions in dogs. 64-72.

Evans, H. E and Fletcher, T. F. 1979. The skeleton and Spinal cord meninges. Miller's Anatomy of the Dog. W.B. Saunders Philadelphia, $3^{\text {rd }}$ Edition, 166, 802-805.

Jones, J. C and D. Inzana, D. 2000. Subclinical CT abnormalities in lumbosacral spine of old large breed dogs. Vet Radiol Ultrasound. 41: 19-26.

Jones, J. C., Cartee, R. E and Bartels, J. E. 1995. Vet Radiol Ultrasound. 36 (2): 91-99.

Kinnis, J., Mai, W., Seiler, Zwingenberger and Schwarz, T. 2006. Radiographic sensitivity and negative predictive value for acute canine spinal trauma. Vet Radiol Ultrasound. 47: 563-570.

Lecouteur, R. A. 2004. World Small Animal Association World Congress Proceeding.

Olby, N. J., Munana, K. R., Sharp, N, J and Thrall, D. E. 2000. The computed tomographic appearance of acute thoracolumbar intervertebral disc herniation in dogs. Vet Radiol Ultrasound. 41: 396-402.

Palus, V. 2014. Neurological examination in small animals. Mac Rev Vet. 37(1): 95105.

Schulz, K.S., Walker, M., Moon, M., Waldron, D., Slater, M and Mcdonald, D. E. 1998. Correlation of clinical radiographic and surgical localization of intervertebral disc extrusion in small breed dogs: A prospective study of 50 cases. Vet Surg. 27: 105-111. 


\section{How to cite this article:}

Heera Banu, S., 2018. Computed Tomographic Diagnosis of Spinal Diseases in Dogs. Int.J.Curr.Microbiol.App.Sci. 7(09): 3618-3621. doi: https://doi.org/10.20546/ijcmas.2018.709.449 